\title{
CINÉTICA DE CAMBIOS SENSORIALES Y VIDA DE ANAQUEL DE CARAMBOLA MÍNIMAMENTE PROCESADA
}

\section{KINETICS OF SENSORY CHANGES AND SHELF-LIFE OF MINIMALLY PROCESSED STAR FRUIT}

\author{
Gladys González-González¹, María E. Pirovani², Andrea M. Piagentini², \\ Fidel Ulín-Montejo ${ }^{3}$, Edith Miranda-Cruz ${ }^{1}$, Rodolfo Osorio-Osorio ${ }^{1}$, Emilio \\ J. Maldonado-Enríquez ${ }^{4}$ y Rosa M. Salinas-Hernández ${ }^{1 *}$
}

\begin{abstract}
'División Académica de Ciencias Agropecuarias, Universidad Juárez Autónoma de Tabasco. Km 25 Carretera Villahermosa-Teapa. 86000, Centro. Tabasco, México. ${ }^{2}$ Instituto de Tecnología de Alimentos, Facultad de Ingeniería Química, Universidad Nacional del Litoral. Santiago del Estero 2829 Santa Fe, Argentina. ${ }^{3}$ División Académica de Ciencias Básicas, Universidad Juárez Autónoma de Tabasco. Km. 1 Carretera Cunduacán-Jalpa. 86690 La Esmeralda,Tabasco, México. ${ }^{4}$ División Académica Multidisciplinaria de los Ríos, Universidad Juárez Autónoma de Tabasco. Km. 1 Carretera Tenosique-Estapilla. 86901, Tenosique, Tabasco, México.
\end{abstract}

\section{RESUMEN}

Con el propósito de establecer condiciones de almacenamiento adecuadas, para reducir la pérdida de calidad en la distribución de carambola mínimamente procesada, frutos de carambola (Averrhoa carambola L.) frescos cortados se almacenaron a $4.9,7.8$ y $12.8{ }^{\circ} \mathrm{C}$ durante 14,8 y $3 \mathrm{~d}$, respectivamente. Las muestras fueron evaluadas por un panel entrenado y la cinética de los cambios sensoriales se determinó mediante el ajuste de modelos de cero y primer orden, con base en el coeficiente de determinación $\left(R^{2}\right)$ obtenido por análisis de regresión lineal simple. A partir de las constantes cinéticas del modelo ajustado, se obtuvo la energía de activación $\left(E_{a}\right)$ para cada atributo, mediante la ecuación de Arrhenius. La vida de anaquel del producto se estimó a partir de las constantes cinéticas y el valor medio de la escala lineal de $10 \mathrm{~cm}$ como valor límite de vida útil, para cada atributo evaluado. Los cambios en olor característico, olor extraño, brillo, sabor característico, consistencia y jugosidad ajustaron mejor a cinéticas de primer orden y el resto a cinéticas de orden cero. Los valores más altos de $E_{a}$ fueron para consistencia, olor característico y sabor extraño (254.87, 225.60 y 214.31 $\mathrm{kJ} \mathrm{mol}^{-1}$, respectivamente), mientras que los más bajos fueron para color característico, jugosidad y olor fermentado $\left(134.18,141.35\right.$ y $\left.141.38 \mathrm{~kJ} \mathrm{~mol}^{-1}\right)$. La vida de anaquel estimada de la carambola mínimamente procesada fue de 11,6 y $2 \mathrm{~d}$ para las temperaturas de $4.9,7.8$ y $12.8^{\circ} \mathrm{C}$, respectivamente. Los valores de $E_{a}$ estimados remarcan la necesidad de un adecuado manejo de temperatura durante el procesamiento, almacenamiento y comercialización del producto. Los cambios que acortan la vida de anaquel fueron la aparición de olor fermentado y oscurecimiento.

Palabras clave: Averrhoa carambola, almacenamiento refrigerado, deterioro de calidad, procesado mínimo, vida útil sensorial.

\section{SUMMARY}

In order to establish appropriate conditions of storage, to reduce losses of quality in the distribution of fresh-cut star fruit (Averrhoa carambola L.), minimally processed fruits were stored at $4.9,7.8$ and $12.8^{\circ} \mathrm{C}$ for 14,8 and $3 \mathrm{~d}$, respectively. The samples were evaluated by a trained panel and kinetics of sensory changes were determined by fitting models of zero and first order, obtained from a deterioration general model, based on the coefficient of determination $\left(R^{2}\right)$ obtained by linear regression analysis. From the kinetic constants of the fitted model, the activation energy $\left(E_{a}\right)$ for each attribute was obtained by means of the Arrhenius equation. The shelf-life of the product was estimated from the kinetic constants and the middle value of the linear scale of $10 \mathrm{~cm}$ as shelf-life limit value, for each attribute evaluated. Changes in aroma, off-odor, brightness, flavor, cohesiveness and juiciness, fitted better to the first-order kinetics and the rest to zero-order kinetics. Greatest values $\left(E_{a}\right)$ were for cohesiveness, off-odor and off-flavor $(254.87,225.60$ y 214.31 $\mathrm{kJ} \mathrm{mol}^{-1}$, respectively), while the lowest values were for color, juiciness and fermented odor $\left(134.18,141.35\right.$ y $\left.141.38 \mathrm{~kJ} \mathrm{~mol}^{-1}\right)$. Shelf-life estimated for the minimally processed star fruit was 11,6 and $2 \mathrm{~d}$ at $4.9,7.8$ and $12.8^{\circ} \mathrm{C}$, respectively. The values estimated of $E_{a}$ highlight the necessity of a proper management of temperature, during the processing, storage and marketing of the product. The changes that limited shelf-life were browning and off-odors.

Index words: Averrhoa carambola, refrigerated storage, quality deterioration, minimal processing, sensory shelf-life.

\section{INTRODUCCIÓN}

El mercado de frutos mínimamente procesados constituye un sector de rápido crecimiento dentro de la industria alimentaria, debido a la alta demanda de alimentos de fácil preparación y consumo que aporten beneficios para la salud (Andrade-Cuvi et al., 2010; Robles-Sánchez et al., 2007). La carambola (Averrhoa carambola L.) es un fruto con potencial para integrarse a este mercado, es nativa de India, Indonesia y Sri Lanka y muy popular en muchos países tropicales (James y Ngarmsak, 2010). Este fruto representa una importante fuente de antioxidantes naturales tales como vitamina C, carotenoides y compuestos fenólicos (Ruiz-López et al., 2011).

El procesamiento mínimo engloba operaciones como lavado, pelado, deshuesado, cortado y sanitizado (Andrade-Cuvi et al., 2010). Posteriormente, los productos son colocados en bolsas selladas o empaques semipermeables y almacenados, con o sin atmósfera modificada, en refrigeración (Francis et al., 2012; Rangel-Marrón y López-Malo, 2012). 
La comercialización de estos productos puede estar limitada en gran parte por excesivo ablandamiento de tejidos y pardeamiento (Teixeira et al., 2012), debido a que después de la cosecha y procesamiento el metabolismo sigue activo (Rangel-Marrón y López-Malo, 2012). Además, el procesado mínimo acelera algunas reacciones biológicas, como la respiración y la pérdida de humedad (Rathod et al., 2011). Estas reacciones inducen a cambios que impactan principalmente las características sensoriales del producto que pueden acortar la vida de anaquel (Alegría et al., 2012; Ruelas-Chacón et al., 2013).

Una herramienta para predecir los cambios que inducen al deterioro y determinan la vida de anaquel en frutos mínimamente procesados es la aplicación de modelos cinéticos de deterioro (García et al., 2011; Salinas-Hernández et al., 2007, 2009). Estos modelos se basan en la cinética de las reacciones químicas (Fu y Labuza, 1997). Sin embargo, hay que considerar que un alimento es un sistema complejo en el que ocurren diferentes tipos de reacciones, por ello la modelación en este caso se aplica, no a un reactante o componente particular del alimento, sino a una característica de calidad que refleja dichas reacciones (SalinasHernández et al., 2007).

De esta manera, según Fu y Labuza (1997), es posible expresar la tasa de pérdida de calidad en función del tiempo, en términos de un pseudo orden u orden aparente de reacción basado en la Ec. 1, donde $A$ es el componente o característica del alimento, a es el orden aparente o pseudo orden de la reacción para el componente $A, k f^{\prime}$ es la constante aparente de reacción, y la notación \pm corresponde al aumento de una característica indeseable $(+)$ y pérdida de un característica deseable (-).

$$
r=\frac{ \pm d[A]}{d t}=k f^{\prime}[A]^{a} \quad \text { EC. (1) }
$$

A partir de la Ec. 1 se obtiene la ecuación general de deterioro de la calidad (Ec. 2) propuesta por Fu y Labuza (1997), donde $Q$ es el atributo de calidad, $t$ es tiempo (d), $n$ es orden de reacción y $k$ es constante aparente de reacción. El signo $(+)$ hace referencia a atributos cuyo valor aumenta con el tiempo y el signo (-) a atributos cuyo valor disminuye con el tiempo.

$$
\pm \frac{d Q}{d t}=k Q^{n} \quad \text { Ec. (2) }
$$

Asimismo, para describir la dependencia de la constante aparente de velocidad de cambio respecto a la temperatura se utilizó la ecuación de Arrhenius (Ec. 3), donde $K$ es la constante cinética de Arrhenius, $k_{0}$ es un factor preexponencial, $E_{a}$ es energía de activación $\left(\mathrm{J} \mathrm{mol}^{-1}\right), R$ es la constante de los gases ideales (8.31447 $\mathrm{J} \mathrm{K}^{-1} \mathrm{~mol}^{-1}$ ) y $T$ es temperatura (K).

$$
K_{T}=K_{0} \exp \left(-\left(\frac{E_{a}}{R T}\right)\right) \quad \text { Ec. (3) }
$$

Nelson y Labuza (1994) obtuvieron que el modelo de Arrhenius es útil para describir la dependencia, respecto a la temperatura, de las constantes de reacción en el intervalo en que la mayoría de los alimentos son almacenados (de -20 a $0{ }^{\circ} \mathrm{C}$ para alimentos congelados y de 4 a $45^{\circ} \mathrm{C}$ para alimentos refrigerados o deshidratados).

El objetivo de este trabajo fue aplicar modelos cinéticos predictivos de la pérdida de calidad en función del tiempo y la temperatura, lo que puede permitir proponer condiciones de conservación adecuadas para reducir las pérdidas de calidad en la distribución de carambola mínimamente procesada.

\section{MATERIALES Y MÉTODOS}

\section{Obtención de los frutos y preparación de muestras}

Frutos de carambola tipo ácida (Mateus-Cagua et al., 2015), en madurez de consumo, coloración amarilla uniforme y ausencia de daños físicos aparentes, fueron cosechadas a 17 km del laboratorio, en la Finca "Las Lilias", ubicada en el km 42 de la Carretera Villahermosa-Teapa, Tabasco, México (170 40'14.0" N, $92^{\circ} 56^{\prime} 57.9^{\prime \prime}$ O) y transportados inmediatamente al laboratorio para su procesamiento. La madurez del fruto al momento del proceso fue caracterizada mediante la evaluación de sólidos solubles (SST), pH, acidez titulable y color (Cuadro 1). El rendimiento en fruto procesado fue de $64.8 \%$ por lo que en cada procesamiento y para cada temperatura de evaluación se utilizaron 10 frutos. El procesamiento se realizó en intervalos de 48, 24 y $10 \mathrm{~h}$ para las temperaturas de $4.9,7.8$ y $12.8^{\circ} \mathrm{C}$, respectivamente, para obtener siete puntos de evaluación en cada caso.

Durante el experimento se cosecharon cuatro lotes de frutos, los cuales fueron conservados a $12{ }^{\circ} \mathrm{C}$ por un máximo de $3.5 \mathrm{~d}$ hasta el momento del proceso. Los frutos fueron lavados con agua corriente, sanitizados en agua clorada (200 $\left.\mathrm{mg} \mathrm{L}^{-1}\right)$ y secados sobre papel absorbente. Posteriormente, se cortaron manualmente en rebanadas en forma de estrella de 5 a $7 \mathrm{~mm}$ de espesor aproximadamente, y se retiró la cáscara y semillas. El producto se sanitizó en agua clorada ( $80 \mathrm{mg} \mathrm{L}^{-1}$ ) y el resto de la solución sanitizante se eliminó por centrifugado con equipo manual para vegetales durante $20 \mathrm{~s}$ ( 2 ciclos s-1).

El envasado se realizó en recipientes de poliestireno con tapa (30 g por muestra) y el almacenamiento fue en cámaras refrigeradas con regulador de temperatura. El monitoreo de la temperatura durante el almacenamiento indicó que las 
Cuadro 1. Características fisicoquímicas de frutos de carambola cosechados en madurez de consumo

\begin{tabular}{|c|c|c|c|c|}
\hline \multirow{2}{*}{ Características } & \multicolumn{4}{|c|}{ Momento de cosecha } \\
\hline & 1 & 2 & 3 & 4 \\
\hline Firmeza (N) & $3.0 \pm 0.9$ & $2.8 \pm 1.0$ & $2.9 \pm 1.0$ & $2.5 \pm 0.3$ \\
\hline Sólidos solubles ( ${ }^{\circ} \mathrm{Brix}$ ) & $5.0 \pm 0.1$ & $5.1 \pm 0.3$ & $5.8 \pm 0.4$ & $5.5 \pm 0.9$ \\
\hline $\mathrm{pH}$ & $2.2 \pm 0.1$ & $2.6 \pm 0.1$ & $3.2 \pm 0.8$ & $2.6 \pm 0.3$ \\
\hline Acidez titulable (\% ac. oxálico) & $0.32 \pm 0.01$ & $0.26 \pm 0.01$ & $0.26 \pm 0.03$ & $0.27 \pm 0.03$ \\
\hline \multicolumn{5}{|l|}{ Parámetros de color } \\
\hline$L^{*}$ & $57.9 \pm 1.5$ & $52.3 \pm 2.2$ & $59.1 \pm 5.2$ & $56.3 \pm 5.9$ \\
\hline$a^{\star}$ & $3.7 \pm 0.9$ & $5.0 \pm 0.7$ & $5.5 \pm 2.8$ & $5.2 \pm 1.1$ \\
\hline$b^{\star}$ & $38.2 \pm 3.1$ & $42.1 \pm 2.5$ & $41.4 \pm 9.0$ & $41.7 \pm 7.0$ \\
\hline$C^{*}$ & $38.4 \pm 3.1$ & $42.4 \pm 2.4$ & $41.8 \pm 9.3$ & $42.0 \pm 7.1$ \\
\hline$h^{0}$ & $84.4 \pm 1.4$ & $83.2 \pm 1.3$ & $82.8 \pm 2.0$ & $82.9 \pm 0.5$ \\
\hline
\end{tabular}

$\mathrm{L}^{\star}=$ componente del color correspondiente a la luminosidad (de 0 a 100); $\mathrm{a}^{\star}=$ componente del color que indica variación entre rojo $(+a)$ y verde $(-a) ; b^{*}=$ componente del color que indica variación entre amarillo $(+b)$ y azul $(-b)$; $C^{\star}=$ Croma, indica intensidad de color $\left(C^{\star}=\left(a^{\star 2}+b^{\star 2}\right)^{1 / 2}\right) ; h^{0}=$ ángulo de matiz, hace referencia a la percepción del color de forma similar a la del ojo humano $\left(h^{\circ}=\operatorname{arctg}\left(b^{*} / a^{*}\right)\right)$.

temperaturas promedio a las que se expuso el producto fueron $4.9,7.8$ y $12.8^{\circ} \mathrm{C}$, durante 14,8 y 3 d, respectivamente. Estas temperaturas se seleccionaron con el objetivo de representar tres tipos de almacenamiento en refrigeración: industrial, comercial y con ligero abuso de temperatura en punto de venta u hogar.

\section{Entrenamiento del panel para evaluación sensorial}

Los jueces que integraron el panel sensorial habían sido previamente preseleccionados con base en su interés, disponibilidad, estado de salud y hábitos alimenticios, y luego evaluados en cuanto a su habilidad en la detección e identificación de olores y en su capacidad discriminante de los atributos sensoriales de color, dulzor, acidez y textura evaluados sobre muestras estándar (AENOR, 1997). La selección final de jueces se hizo con base en los resultados de un análisis secuencial (Meilgaard et al., 1999) aplicado a los resultados de las pruebas discriminativas realizadas.

Para el experimento, se prepararon muestras con diferente grado de deterioro para el reconocimiento y evaluación por parte de los jueces, quienes generaron una lista individual de términos descriptores del deterioro del producto (Salinas-Hernández et al., 2010). La selección final de descriptores para integrar la planilla de evaluación se hizo con el método reportado por Moskowitz (1983). Una vez generada la planilla, se procedió al entrenamiento y calibración del panel.

Para cada sesión de entrenamiento los jueces evaluaron muestras de referencia del producto (con diferentes tiempos de almacenamiento), y los resultados de cada sesión se analizaron mediante análisis de varianza (ANOVA) para un diseño completamente al azar con arreglo factorial, donde los factores fueron el tiempo de almacenamiento y los jueces sensoriales (Salinas-Hernández et al., 2010). El entrenamiento concluyó al no observar diferencias significativas entre 10 de los 12 panelistas, cinco hombres y cinco mujeres, con quienes se procedió a la evaluación de las muestras. La planilla de evaluación quedó conformada por descriptores correspondientes a la apariencia, olor, sabor y textura. Los descriptores, definiciones y términos ancla se muestran en el Cuadro 2.

\section{Evaluación sensorial}

Las muestras del experimento se obtuvieron mediante un diseño experimental escalonado (Gámbaro, 2005) y fueron evaluadas en cabinas individuales, un día específico para cada temperatura experimental. Los jueces recibieron las muestras en forma aleatoria y en su recipiente original, previamente codificadas con números aleatorios de tres cifras, y la intensidad con la que cada juez percibió cada uno de los atributos fue indicada colocando una marca en una escala lineal no estructurada, es decir sin puntos de referencia intermedios, de manera que el juez indicó su apreciación de la intensidad del estímulo en la línea que une los extremos de la escala, donde se colocan los términos ancla correspondientes a la intensidad mínima y máxima del estímulo evaluado (Salinas-Hernández et al., 2010; Salinas-Hernández et al., 2015). La escala utilizada fue de 
Cuadro 2. Características sensoriales y descriptores utilizados en la evaluación de carambola mínimamente procesada.

\begin{tabular}{|c|c|c|c|}
\hline Características sensoriales & Descriptores & Definición & Términos ancla \\
\hline \multirow[t]{5}{*}{ Apariencia } & Apariencia & $\begin{array}{l}\text { Relativo al color, forma, aspecto, integridad, homo- } \\
\text { geneidad y ausencia de defectos. }\end{array}$ & Pobre - óptima \\
\hline & Frescura* & $\begin{array}{l}\text { Relativo a la condición de estar fresco o lo más } \\
\text { próximo a la cosecha. Caracterizado por una mayor } \\
\text { turgencia de las células y colores vivos. }\end{array}$ & Pobre - óptima \\
\hline & Brillo & $\begin{array}{l}\text { Relativo a la luminosidad relacionada con la forma } \\
\text { en que la luz interactúa con la superficie del pro- } \\
\text { ducto. Término contrario a opacidad. La humedad } \\
\text { superficial y la viveza del color determinan el brillo } \\
\text { o bien la opacidad. }\end{array}$ & Opaco -brillante \\
\hline & Color característico & $\begin{array}{l}\text { Color amarillo-naranja intenso, asociado al fruto de } \\
\text { carambola en madurez de consumo. }\end{array}$ & Pobre - óptimo \\
\hline & Oscurecimiento & $\begin{array}{l}\text { Relativo a la presencia de color oscuro o pardo en } \\
\text { las superficies de corte del producto. }\end{array}$ & Nulo - intenso \\
\hline \multirow[t]{3}{*}{ Olor } & Olor característico & $\begin{array}{l}\text { Asociado a las notas propias del fruto de carambo- } \\
\text { la fresco y recién cortado. }\end{array}$ & Pobre - óptimo \\
\hline & Olor extraño & $\begin{array}{l}\text { Olor no característico del fruto de carambola recién } \\
\text { cortado }\end{array}$ & Nulo - intenso \\
\hline & Olor fermentado* & Olor asociado con frutos o vegetales fermentados. & Nulo - intenso \\
\hline \multirow[t]{2}{*}{ Sabor } & Sabor característico & $\begin{array}{l}\text { Término general asociado a las notas propias del } \\
\text { fruto de carambola. }\end{array}$ & Pobre - óptimo \\
\hline & Sabor extraño & $\begin{array}{l}\text { Sabor no característico del fruto de carambola re- } \\
\text { cién cortado }\end{array}$ & Nulo - intenso \\
\hline \multirow[t]{3}{*}{ Textura } & Consistencia* & $\begin{array}{l}\text { Relacionada con el grado de compactación y resis- } \\
\text { tencia a la deformación y al corte, de los tejidos del } \\
\text { fruto, percibido al morder y masticar. }\end{array}$ & Blanda - firme \\
\hline & Jugosidad* & $\begin{array}{l}\text { Relativo a la cantidad de jugo/humedad percibida } \\
\text { en la boca. }\end{array}$ & Nula - intensa \\
\hline & Textura fibrosa & $\begin{array}{l}\text { Relativo a la presencia de fibras vegetales que in- } \\
\text { fluyen en la fuerza y número de masticadas nece- } \\
\text { sarias para preparar la muestra, en la boca, para su } \\
\text { deglución. }\end{array}$ & Nula - intensa \\
\hline
\end{tabular}

Fuente: *Adaptado de Bett (2002) y elaboración propia.

$10 \mathrm{~cm}$ y los términos ancla utilizados en cada atributo se muestran en el Cuadro 2.

En general el término ancla del extremo izquierdo corresponde a una baja o nula intensidad en el atributo a evaluar, y el ancla derecha a la intensidad máxima. La intensidad máxima y mínima, en cada atributo, fue determinada durante el entrenamiento sobre las muestras de referencia.

Los resultados se obtuvieron al medir la distancia $(\mathrm{cm})$ desde el ancla izquierda $(0 \mathrm{~cm})$ hasta la marca colocada sobre la escala por el evaluador, correspondiente a la intensidad percibida del atributo en cuestión.

\section{Determinación de la cinética de los cambios sensoriales}

Para determinar la cinética de cambio de los atributos sensoriales, se utilizó la Ec. (2) general de deterioro (Fu y Labuza, 1997; Salinas-Hernández et al., 2015). Se evaluaron dos tipos de cinéticas, una de orden cero y la otra de primer orden (Amodio et al., 2013; Salinas-Hernández et al., 2009). Para el primer caso se consideró a $n$ con valor igual a cero y para el segundo con valor igual a 1. Por lo tanto, al integrar la Ec. (2) se obtuvieron las ecuaciones correspondientes a estas cinéticas de cambio. Para una reacción de orden cero $(n=0)$ la expresión correspondiente es la Ec. (4), en tanto que cuando la reacción es de primer orden $(n=1)$ la cinética se expresa en forma de la Ec. (5). 


$$
\begin{aligned}
& \pm \frac{d Q}{d t}=k \\
& \pm \frac{d Q}{d t}=k Q
\end{aligned}
$$

Después de resolver las Ec. (4) y (5), se obtuvieron las expresiones correspondientes para las cinéticas de orden cero (Ec. 6) y primer orden (Ec. 7), donde $Q_{0}$ es valor inicial del atributo de calidad ( $\mathrm{cm}), Q$ el valor del atributo en el tiempo $t(\mathrm{~cm}), k$ es la constante aparente de reacción y $t$ es tiempo $(d)$

$$
\begin{array}{ll}
Q=Q_{0} \pm k t & \text { Ec. (6) } \\
Q=Q_{0} e^{ \pm k t} & \text { Ec. (7) }
\end{array}
$$

Con base en lo anterior, la cinética de cambio para cada atributo sensorial se determinó mediante el ajuste de los datos experimentales a los modelos de cero y primer orden, mediante el procedimiento de regresión lineal a los datos sensoriales, por medio de Office Microsoft Excel® 2013.

\section{Dependencia de la temperatura}

Para describir la dependencia de la constante aparente de velocidad de cambio, respecto a la temperatura, se utilizó la ecuación de Arrhenius (Ec. 3) en su forma linealizada (Ec. 8), donde $k$ es la constante aparente de reacción, $E_{a}$ es la energía de activación $\left(\mathrm{J} \mathrm{mol}^{-1}\right), R$ es la constante de los gases ideales (8.31447 $\left.\mathrm{J} \mathrm{K}^{-1} \mathrm{~mol}^{-1}\right), T$ es temperatura $(\mathrm{K})$ y $K_{0}$ es el factor pre-exponencial.

$$
\ln k=\frac{-E_{a}}{R} \frac{1}{T}+\ln k_{0} \quad \text { EC. (8) }
$$

Para obtener el valor estimado de la energía de activación $\left(E_{a}\right)$ para cada atributo, se aplicó el procedimiento de regresión lineal, esta vez a los valores de la constante aparente de reacción obtenidos a diferentes temperaturas evaluadas en el experimento.

\section{Predicción de la vida de anaquel}

La vida de anaquel de carambola mínimamente procesada a cada temperatura de almacenamiento, se estimó a partir de la Ec. (9) (Salinas-Hernández et al., 2015), donde $Q_{0}$ es el valor inicial del atributo de calidad, $Q_{e}$ es el valor alcanzado del atributo al tiempo $t_{s^{\prime}}$ donde $t_{s}$ es el tiempo final de la vida de anaquel del producto y $k$ la constante aparente de reacción.

$$
Q_{e}=Q_{0}-k t_{s} \quad \text { Ec. }(9)
$$

A partir de la Ec. (9), una vez establecido el valor límite de vida útil para el atributo o característica en cuestión, la vida de anaquel para las cinéticas de cambio de cero y primer orden se estimó mediante las Ecs. (10) y (11), respectivamente (Salinas-Hernández et al., 2009).

$$
\begin{aligned}
t_{s} & =\frac{Q_{0}-Q_{e}}{k} \\
t_{s} & =\frac{\ln \frac{Q_{0}}{Q_{e}}}{k}
\end{aligned}
$$

En este trabajo, se estableció el punto medio de la escala lineal de $10 \mathrm{~cm}(5.0 \mathrm{~cm})$ como valor límite de vida útil para cada atributo al final del almacenamiento (Piagentini et al., 2004; Piagentini et al., 2005).

\section{Análisis estadístico}

Se aplicó un análisis de varianza (ANOVA) a los datos obtenidos en la evaluación sensorial para determinar cambios significativos en los atributos evaluados en función del tiempo y temperatura de almacenamiento. El criterio de mejor ajuste de datos experimentales a los modelos cinéticos evaluados fue el coeficiente de determinación $\left(\mathrm{R}^{2}\right)$. Los coeficientes de determinación correspondientes a los modelos de cero y primer orden, para cada atributo, se compararon mediante una prueba t de Student para evaluar diferencias en el ajuste a cada modelo evaluado. Los análisis se realizaron a un nivel de confiabilidad de $95 \%$.

\section{RESULTADOS Y DISCUSIÓN}

De acuerdo con los resultados de análisis de datos sensoriales, los atributos de carambola mínimamente procesada que más disminuyeron fueron textura fibrosa, apariencia y frescura, y los que más aumentaron fueron olor fermentado y oscurecimiento. En el Cuadro 3 se muestran los coeficientes de determinación $\left(R^{2}\right)$ obtenidos del ajuste de los promedios de los datos experimentales a los modelos cinéticos de cero y primer orden. Los resultados muestran que los cambios en olor característico, olor extraño, brillo, sabor característico, consistencia y jugosidad, se ajustaron mejor a cinéticas de primer orden, mientras que el resto de los atributos siguieron una cinética de orden cero.

En las Figuras 1 y 2 se muestra la tendencia de los datos experimentales y de los estimados con el modelo de mejor ajuste, donde se puede observar la efectividad de la aplicación de estos modelos para describir los cambios en atributos sensoriales del fruto evaluado. Estos resultados son congruentes con los obtenidos en estudios previos, donde se ha demostrado un buen ajuste de los cambios sensoriales de productos vegetales mínimamente procesados, a cinéticas de orden cero y primer orden (Amodio et al., 2013; 
Cuadro 3. Coeficientes de determinación $\left(\mathrm{R}^{2}\right)$ de los modelos cinéticos de cero y primer orden para atributos sensoriales de carambola mínimamente procesada.

\begin{tabular}{|c|c|c|c|c|c|c|}
\hline \multirow{3}{*}{ Atributo } & \multicolumn{6}{|c|}{ Temperatura $\left({ }^{\circ} \mathrm{C}\right)$} \\
\hline & \multicolumn{2}{|c|}{4.9} & \multicolumn{2}{|c|}{7.8} & \multicolumn{2}{|c|}{12.8} \\
\hline & $\mathrm{n}=0$ & $\mathrm{n}=1$ & $\mathrm{n}=0$ & $\mathrm{n}=1$ & $\mathrm{n}=0$ & $n=1$ \\
\hline Olor característico & 0.975 & 0.980 & 0.909 & 0.920 & 0.958 & 0.935 \\
\hline Olor fermentado & 0.971 & 0.964 & 0.978 & 0.926 & 0.976 & 0.910 \\
\hline Olor extraño & 0.879 & 0.968 & 0.952 & 0.800 & 0.895 & 0.964 \\
\hline Apariencia & 0.933 & 0.955 & 0.915 & 0.905 & 0.950 & 0.936 \\
\hline Frescura & 0.948 & 0.961 & 0.978 & 0.948 & 0.970 & 0.968 \\
\hline Brillo & 0.882 & 0.907 & 0.941 & 0.961 & 0.965 & 0.966 \\
\hline Color característico & 0.940 & 0.939 & 0.990 & 0.974 & 0.917 & 0.884 \\
\hline Oscurecimiento & 0.977 & 0.876 & 0.927 & 0.785 & 0.955 & 0.826 \\
\hline Sabor característico & 0.967 & 0.983 & 0.952 & 0.964 & 0.876 & 0.731 \\
\hline Sabor extraño & 0.929 & 0.956 & 0.922 & 0.749 & 0.942 & 0.892 \\
\hline Consistencia & 0.959 & 0.962 & 0.977 & 0.988 & 0.977 & 0.891 \\
\hline Jugosidad & 0.985 & 0.982 & 0.972 & 0.981 & 0.929 & 0.933 \\
\hline Textura fibrosa & 0.946 & 0.935 & 0.930 & 0.914 & 0.973 & 0.958 \\
\hline
\end{tabular}

Piagentini et al., 2005; Salinas et al., 2007; Salinas-Hernández et al., 2010).

En el Cuadro 4 se muestran las constantes aparentes de reacción obtenidas a partir del modelo de mejor ajuste a los datos experimentales. Los valores de estas constantes fueron mayores al incrementarse la temperatura, ya que se registró una mayor velocidad de cambio en el atributo con respecto al tiempo (Salinas-Hernández et al., 2009). En el mismo Cuadro 4 se muestran también los valores de la energía de activación $\left(E_{a}\right)$ de los cambios evaluados. La velocidad de cambio en las características sensoriales indicó que los atributos relacionados con el olor y la apariencia tuvieron los mayores valores de las constantes cinéticas durante el almacenamiento. En el caso del olor, el fermentado y el olor extraño mostraron cambios significativos. El olor fermentado tuvo la mayor velocidad de cambio a las tres temperaturas evaluadas.

Para los descriptores de la apariencia la mayor velocidad de cambio se observó en el oscurecimiento, lo que se relaciona con los valores obtenidos también en el caso de la apariencia, la frescura y el color característico del producto (Cuadro 4). Este comportamiento fue consistente con los valores de $E_{a}$ obtenidos. En este sentido, el color característico, la jugosidad, el oscurecimiento y el olor fermentado mostraron la mayor dependencia y, por tanto mayor afectación respecto una variación de la temperatura. De acuerdo con esta información, si se realiza un manejo inadecuado de la temperatura durante la cadena de frío las primeras alteraciones que podrían presentarse en el producto afectarían la apariencia y el olor, debido principalmente al oscurecimiento y aparición de olores extraños, específicamente olor fermentado.

Estos resultados confirman los observados en otros frutos mínimamente procesados, en los cuales los cambios sensoriales, específicamente la apariencia, son las principales limitantes de la vida de anaquel (Fiszman, 2005; Salinas-Hernández et al., 2015). Al respecto, Robles-Sánchez et al. (2007) indicaron que el almacenamiento en frío (de 0 a $5^{\circ} \mathrm{C}$ ), en atmósferas controladas o modificadas y el tratamiento con estabilizadores de color y retenedores de firmeza pueden extender la vida de anaquel hasta por $10 \mathrm{~d}$, sin cambios aparentes en la calidad comercial de los frutos mínimamente procesados. Sin embargo, los estudios sobre mantenimiento de la calidad comercial en frutos tropicales frescos cortados son escasos.

La información aportada por estos datos es consistente con lo mencionado por Ding et al. (2007) y Robles-Sánchez et al. (2007) con relación a que la vida útil de frutos frescos cortados está limitada principalmente por la alteración del color, pérdida de olores y sabores característicos, ablandamiento de los tejidos, así como la generación de olores indeseables, debido al incremento en la actividad metabólica del fruto y la descompartimentalización de enzimas y sustratos a consecuencia de operaciones como el pelado y cortado. 

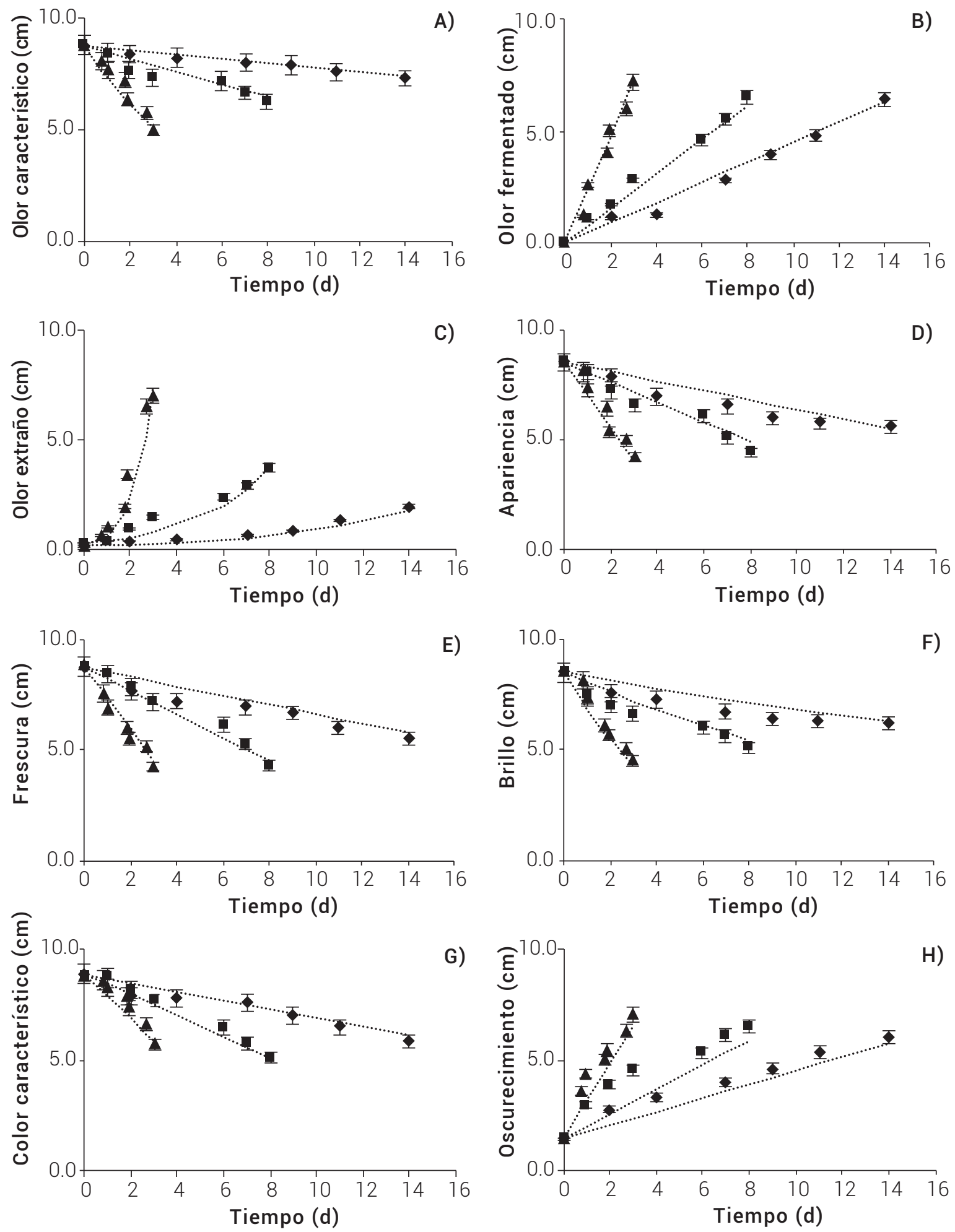

$4.9^{\circ} \mathrm{C} \diamond 7.8^{\circ} \mathrm{C} \square 12.8^{\circ} \mathrm{C} \triangle$ predichos ....

Figura 1. Valores experimentales y predichos de olor característico, olor fermentado, olor extraño, apariencia, frescura, brillo, color característico y oscurecimiento de carambola mínimamente procesada y almacenada a tres temperaturas. 

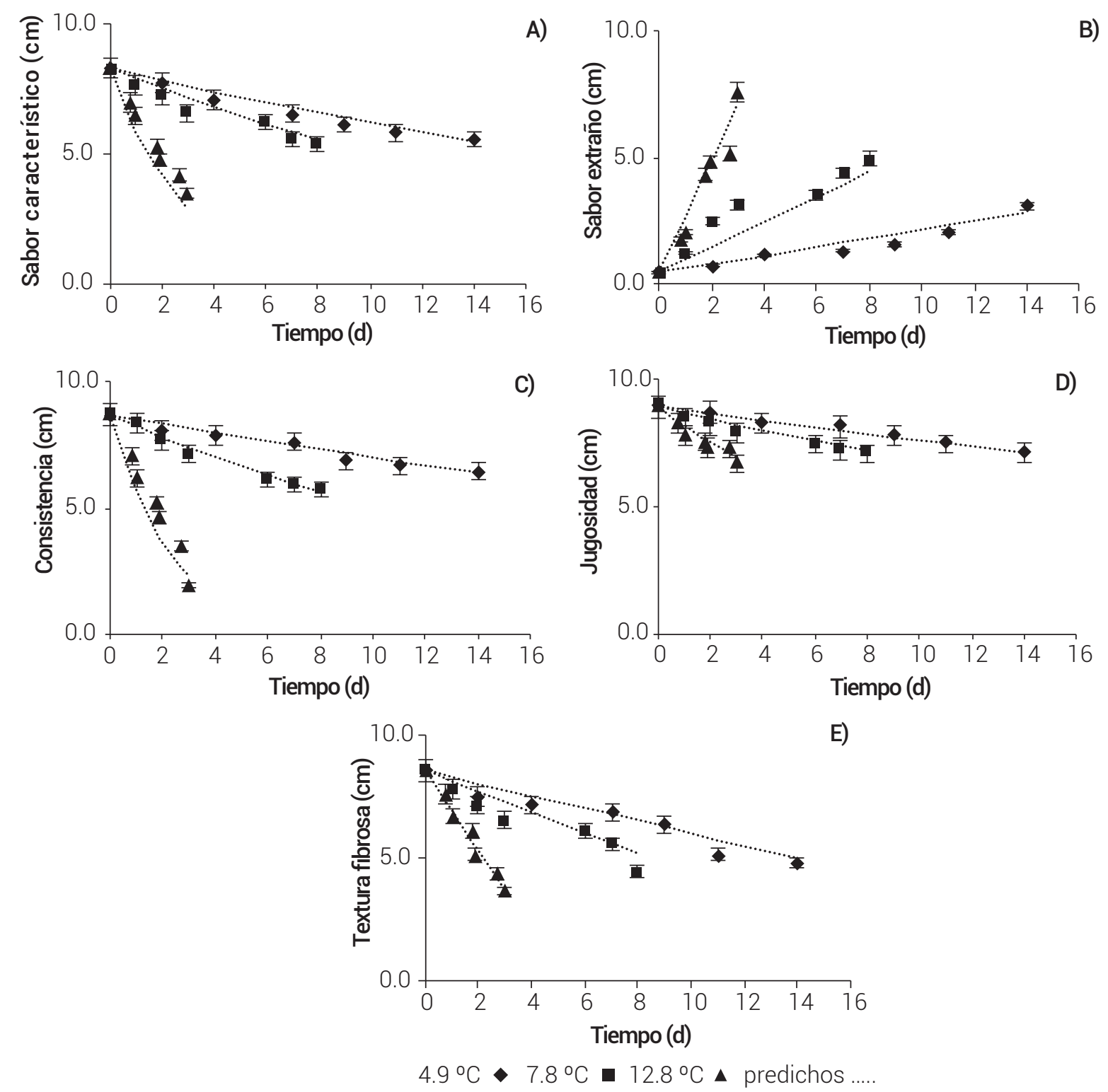

Figura 2. Valores experimentales y predichos de sabor característico, sabor extraño, consistencia, jugosidad y textura fibrosa de carambola mínimamente procesada y almacenada a tres temperaturas.

Los resultados registrados en este experimento indicaron que el incremento en olor fermentado y oscurecimiento fueron los cambios que redujeron la vida de anaquel de carambola mínimamente procesada. En este sentido, algunos autores (Teixeira et al., 2008; Teixeira et al., 2012), mencionan que el oscurecimiento es la principal restricción para el uso de carambola fresca cortada, y que esta reacción es comúnmente asociada a la oxidación enzimática de sustratos fenólicos presentes en el fruto. Finalmente, en función de los cambios limitantes identificados la vida de anaquel de carambola mínimamente procesada y almacenada a $4.9,7.8$ y $12.8^{\circ} \mathrm{C}$, fue de 11,6 y $2 \mathrm{~d}$, respectivamente.
Por lo tanto, el almacenamiento del producto a temperaturas cercanas a $4.9^{\circ} \mathrm{C}$, es recomendable para una mejor preservación de sus atributos sensoriales e incremento de la vida de anaquel.

\section{CONCLUSIONES}

Los modelos cinéticos de cero y primer orden permitieron estimar los cambios en la calidad sensorial de carambola mínimamente procesada y almacenada a temperaturas en el rango de 4.9 y $12.8^{\circ} \mathrm{C}$. Los resultados observados son de utilidad para establecer estrategias adecuadas de 
Cuadro 4. Constantes de reacción ( $k$ y energías de activación $\left(E_{a}\right)$ de la cinética de cambio $(n)$ de atributos sensoriales de carambola mínimamente procesada.

\begin{tabular}{|c|c|c|c|c|c|c|}
\hline \multirow{2}{*}{ Atributo } & \multirow{2}{*}{$\mathrm{n}$} & \multicolumn{3}{|c|}{$k$} & \multirow{2}{*}{$E_{a}\left(\mathrm{KJ} \mathrm{mol}^{-1}\right)$} & \multirow{2}{*}{$* \mathrm{R}^{2}$} \\
\hline & & $4.9^{\circ} \mathrm{C}$ & $7.8^{\circ} \mathrm{C}$ & $12.8^{\circ} \mathrm{C}$ & & \\
\hline Olor característico & 1 & 0.0120 & 0.0377 & 0.1817 & 225.60 & 0.997 \\
\hline Olor fermentado & 0 & 0.4519 & 0.7689 & 2.4103 & 141.38 & 0.996 \\
\hline Olor extraño & 1 & 0.1619 & 0.3174 & 1.2046 & 169.00 & 0.998 \\
\hline Apariencia & 0 & 0.2153 & 0.4503 & 1.5029 & 162.43 & 0.999 \\
\hline Frescura & 0 & 0.2088 & 0.5283 & 1.4059 & 156.60 & 0.983 \\
\hline Brillo & 1 & 0.0219 & 0.0551 & 0.2211 & 192.66 & 0.999 \\
\hline Color característico & 0 & 0.1953 & 0.4757 & 1.0156 & 134.18 & .0 .964 \\
\hline Oscurecimiento & 0 & 0.3099 & 0.5555 & 1.7078 & 143.56 & 0.998 \\
\hline Sabor característico & 1 & 0.0294 & 0.0504 & 0.3456 & 211.48 & 0.972 \\
\hline Sabor extraño & 0 & 0.1688 & 0.4988 & 2.2292 & 214.31 & 0.997 \\
\hline Consistencia & 1 & 0.0216 & 0.0537 & 0.4384 & 254.87 & 0.994 \\
\hline Jugosidad & 1 & 0.0160 & 0.0272 & 0.0853 & 141.35 & 0.996 \\
\hline Textura fibrosa & 0 & 0.2562 & 0.4260 & 1.6219 & 156.92 & 0.988 \\
\hline
\end{tabular}

* $\mathrm{R}^{2}$ es coeficiente de determinación de la regresión del In $\mathrm{k}$ en función de la inversa de temperatura (K).

manejo en la cadena de distribución, con base en la vida de anaquel estimada a partir de estos modelos. Los valores de $E_{a}$ obtenidos para los cambios evaluados indican una fuerte dependencia de la velocidad de éstos con la temperatura, lo cual remarca la necesidad de un adecuado manejo de temperatura durante el procesamiento, almacenamiento y comercialización del producto.

\section{AGRADECIMIENTOS}

Al Consejo Nacional de Ciencia y Tecnología (CONACYT) por la beca otorgada para realizar estudios de posgrado. A los integrantes del panel sensorial de la División Académica de Ciencias Agropecuarias de la UJAT por su apoyo y compromiso en las evaluaciones correspondientes a esta investigación.

\section{BIBLIOGRAFÍA}

AENOR, Asociación Española de Normalización y Certificación (1997) Análisis Sensorial. Asociación Española de Normalización y Certificación. Tomo 1: Alimentación. Recopilación de normas UNE. AENOR, Madrid, España. 253 p.

Alegría C., J. Pinheiro, M. Duthoit, E. M. Goncalves, M. Moldão-Martins and M. Abreu (2012) Freshcut carrot (cv. Nantes) quality as affected by abiotic stress (heat shock and UV-C irradiation) pretreatments. LWT-Food Science and Technology 48:197-203.

Amodio M. L., A. Derossi and G. Colelli (2013) Modelling sensorial and nutritional changes to better define quality and shelf life of freshcut melons. Journal of Agricultural Engineering 44:38-45.

Andrade-Cuvi M. J., C. Moreno-Guerrero, A. Henriquez-Bucheli, A. Gómez-Gordillo y A. Concellón (2010) Influencia de la radiación UV-C como tratamiento postcosecha sobre carambola (Averrhoa carambola L.) mínimamente procesada almacenada en refrigeración.
Revista Iberoamericana de Tecnología Postcosecha 11:18-27.

Bett K. L. (2002) Evaluating Sensory Quality of Fresh-cut Fruits and Vegetables. In: Fresh-cut Fruits and Vegetables: Science, Technology, and Market. O. Lamikanra (ed.). CRC PRESS, Boca Raton, Fl. USA. pp:432-443.

Ding P., S. H. Ahmad and H. M. Ghazali (2007) Changes in selected quality characteristics of minimally processed carambola (Averrhoa carambola L.) when treated with ascorbic acid. Journal of de Science of Food and Agriculture 87:702-709.

Fiszman S. (2005) Análisis sensorial aplicado a la evaluación de las frutas y hortalizas cortadas. In: Nuevas Tecnologías de Conservación de Productos Vegetales Frescos Cortados. G. A. González A. y F. Cuamea-Navarro (eds.). CIAD, Sonora, México. pp:523537.

Francis G. A., A. Gallones, G. J. Nychas, J. N. Sofos, G. Colelli, M. L. Amodio and G. Spano (2012) Factors Affecting Quality and Safety of Fresh-Cut Produce. Critical Reviews in Food Science and Nutrition 52:595-610.

Gámbaro A. (2005) Diseño de ensayos de vida útil de alimentos. In: Estimación de la Vida Útil Sensorial de los Alimentos. G. Hough y S. Fiszman (eds.). CYTED, Madrid, España. pp:43-51.

García B. C., V. G. Chacón y M. E. Molina (2011) Evaluación de la vida útil de una pasta de tomate mediante pruebas aceleradas por temperatura. Ingeniería 21:31-38

James J. and T. Ngarmsak (2010) Processing of Fresh-Cut Tropical Fruits and Vegetables: A Technical Guide. Bangkok, Thailand: FAO Re gional Office for Asia and the Pacific. 102 p.

Fu B. and T. P. Labuza (1997) Shelf-life testing: procedures and prediction methods. Quality in Frozen Food. Chapman \& Hall. New York, USA. pp: 377-415.

Mateus-Cagua D., M. E. Arias y J. O. Orduz-Rodríguez (2015) El cultivo de carambolo (Averrhoa carambola L.) y su comportamiento en el piedemonte del Meta (Colombia). Una revisión. Revista Colombiana de Ciencias Hortícolas 9:135-148.

Meilgaard M., G. V. Civille and B. T. Carr (1999) Selection and training of panel members. In: Sensory Evaluation Techniques 3a ed. CRC Press. Boca Raton, Fla. USA. pp:174-176.

Moskowitz H. R. (1983) Product Testing and Sensory Evaluation of Foods: Marketing and R\&D Approaches. Food \& Nutrition Press, Inc. Trumbull, Conn., USA. 605 p. 
Nelson K. and T. P. Labuza (1994) Water activity and food polymer science: Implications state on Arrhenius and WLF models in predicting shelf life. Journal of Food Engineering 22:271-290

Piagentini A. M., J. C. Méndez, D. R. Güemes and M. E. Pirovani (2005) Modeling changes of sensory attributes for individual and mixed fresh-cut leafy vegetables. Postharvest Biology and Technology 38:202-212.

Piagentini A. M., M. E. Pirovani y D. R. Güemes (2004) Cinética de deterioro de la calidad de repollo fresco cortado. Ciencia y Tecnología Alimentaria 4:169-176.

Rangel-Marrón M. y A. López-Malo (2012) Cambios en frutas tropicales frescas, cortadas y empacadas en atmósfera modificada durante su almacenamiento en refrigeración. Temas Selectos de Ingeniería de Alimentos 6:94-109.

Rathod A., H. Shoba and D. V. Chidanand (2011) A study on shelf life extension of carambola fruits. International Journal of Scientific \& Engineering Research 2:1-5.

Robles-Sánchez M., S. Gorinstein, 0. Martín-Belloso, H. Astiazarán-García, G. González-Aguilar y R. Cruz-Valenzuela (2007) Frutos tropicales mínimamente procesados: potencial antioxidante y su impacto en la salud. Interciencia 32:227-232.

Ruelas-Chacón X., M. Reyes-Vega, B. Valdivia-Urdiales, J. C. ContrerasEsquivel, J. C. Montañez-Saenz, A. F. Aguilera-Carbó y R. D. PeraltaRodríguez (2013) Conservación de frutas y hortalizas frescas y mínimamente procesadas con recubrimientos comestibles. Revista Científica de la Universidad Autónoma de Coahuila 5:31-37.

Ruiz-López I. I., H. Ruiz-Espinosa, E. Herman-Lara and G. Zárate-Castillo (2011) Modeling of kinetics, equilibrium and distribution data of osmotically dehydrated carambola (Averrhoa carambola L.) in sugar solutions. Journal of Food Engineering 104:218-226.
Salinas-Hernández R. M., G. A. González-Aguilar, M. E. Pirovani y F. UlínMontejo (2007) Modelación del deterioro de productos vegetales frescos cortados. Universidad y Ciencia Trópico Húmedo 23:183-197.

Salinas-Hernández R. M., G. A. González-Aguilar and M. E. Tiznado-Hernández (2015) Utilization of physicochemical variables developed from changes in sensory attributes and consumer acceptability to predict the shelf life of fresh-cut mango fruit. Journal of Food Science and Technology 52:63-77

Salinas-Hernández R. M., M. E. Pirovani, A. A. Gardea-Béjar y G. A. GonzálezAquilar (2010) Cambios fisicoquímicos y sensoriales limitantes de la vida de anaquel de mango fresco cortado. Revista Fitotecnia Mexicana 33:215-223

Salinas-Hernández R. M., M. E. Pirovani, F. Ulín-Montejo y G. A. González-Agui$\operatorname{lar}(2009)$ ¿Se puede predecir la vida de anaquel de un alimento? El caso de frutos mínimamente procesados. Revista Industria Alimentaria 31:25-34.

Salinas H. R., S. Reyes, N. Sabbag, S. Acosta, G. González y M. E. Pirovani (2007) Modelación de los cambios en los atributos sensoriales de fresas frescas cortadas. In: $\vee$ Congreso Iberoamericano de Tecnología Postcosecha y Agroexportaciones. Grupo de Postrecolección y Refrigeración. UPCT y Asociación Iberoamericana de Tecnología Postcosecha (eds.). Universidad Politécnica de Cartagena, 29 de mayo de 2007. pp:760-769.

Teixeira G. H. A., J. F. Durigan, R. E. Alves and T. J. O'Hare (2008) Response of minimally processed carambola to chemical treatments and low-oxygen atmospheres. Postharvest Biology and Technology 48:415-421.

Teixeira G. H. A., J. F. Durigan, A. S. Ferraudo, R. E. Alves and T. J. O'Hare (2012) Multivariate analysis of fresh-cut carambola slices stored under different temperature. Postharvest Biology and Technology 63:91-97 